\title{
robustica: customizable robust independent component analysis
}

Miquel Anglada-Girotto ${ }^{1}$, Samuel Miravet-Verde ${ }^{1}$, Luis Serrano ${ }^{1,2,3 *}$, Sarah A. Head ${ }^{1 *}$

\section{Affiliations:}

${ }^{1}$ Centre for Genomic Regulation (CRG), The Barcelona Institute of Science and Technology, Barcelona, Spain.

${ }^{2}$ Universitat Pompeu Fabra (UPF), Barcelona, Spain.

${ }^{3}$ ICREA, Pg. LLuís Companys 23, Barcelona 08010, Spain.

*Corresponding authors: luis.serrano@crg.eu and dibartolosa@gmail.com 


\section{ABSTRACT}

\section{Motivation}

Independent Component Analysis (ICA) allows the dissection of omic datasets into modules that help to interpret global molecular signatures. The inherent randomness of this algorithm can be overcome by clustering many iterations of ICA together to obtain robust components. Existing algorithms for robust ICA are dependent on the choice of clustering method and on computing a potentially biased and large Pearson distance matrix.

\section{Results}

We present robustica, a Python-based package to compute robust independent components with a fully customizable clustering algorithm and distance metric. Here, we exploited its customizability to revisit and optimize robust ICA systematically. From the 6 popular clustering algorithms considered, DBSCAN performed the best at clustering independent components across ICA iterations. After confirming the bias introduced with Pearson distances, we created a subroutine that infers and corrects the components' signs across ICA iterations to enable using Euclidean distance. Our subroutine effectively corrected the bias while simultaneously increasing the precision, robustness, and memory efficiency of the algorithm. Finally, we show the applicability of robustica by dissecting over 500 tumor samples from low-grade glioma (LGG) patients, where we define a new gene expression module with the key modulators of tumor aggressiveness downregulated upon IDH1 mutation.

\section{Availability and implementation}

robustica is written in Python under the open-source BSD 3-Clause license. The source code and documentation are freely available at https://github.com/CRG-CNAG/robustica. Additionally, all scripts to reproduce the work presented are available at https://github.com/MiqG/publication_robustica.

\section{Contact: miquel.anglada@.crg.eu}

\section{Keywords}

bioinformatics; independent component analysis; clustering; unsupervised learning; low-grade glioma; Python. 


\section{INTRODUCTION}

Independent Component Analysis (ICA) is a matrix factorization method that dissects a mixture of signals into a predefined number of additive independent sources or components. ICA finds sets of statistically independent components by minimizing their mutual information ${ }^{1}$. In biology, ICA has a wide range of applications such as defining functional modules, removing technical noise, feature engineering, unsupervised cell type deconvolution, single-cell trajectory inference, or multi-omic analysis (reviewed in ${ }^{2}$ ). Thanks to its information-theoretic objective function, ICA results in components that provide a simpler, more reproducible, and more biologically relevant interpretation than other popular matrix factorization methods such as Principal Component Analysis (PCA) or Non-negative Matrix Factorization (NMF) ${ }^{2-7}$.

Fast $/ C A^{8}$, one of the most widespread algorithms used to perform ICA, starts with a random initialization to decompose the data matrix into a source matrix and a mixing matrix of non-Gaussian independent components (Sup. Fig. 1A). In 2003, Hymberg and Hyvärinen ${ }^{9}$ developed Icasso to address the inherent randomness of Fast/CA, by running Fast/CA multiple times and clustering the components of source matrices across all runs (Sup. Fig. 1B). This clustering step involves two key choices affecting its computational efficiency and the final robust components: the distance metric and the clustering algorithm. Current implementations require pre-computing a potentially large Pearson distance square matrix to cluster components across ICA runs regardless of their different sign and order resulting from Fast/CA's randomness ${ }^{9-12}$. However, correlation-based metrics are sensitive to outliers and non-Gaussian distributions as independent components, which may lead to calculating imprecise weights $^{13}$. Additionally, more recently developed clustering algorithms could potentially improve the efficiency and quality of robust ICA.

Here, we developed robustica, the first Python package to carry out robust ICA with a fully customizable clustering metric and algorithm based on the powerful library scikit-learn ${ }^{14}$. By leveraging the customizability of our package to revisit and optimize the clustering step of the Icasso algorithm, we improved its precision, robustness, and memory efficiency. Finally, as a case study, we dissected gene expression signatures from patients with low-grade glioma (LGG) and found a set of genes related to the mechanism by which mutations in IDH1 lead to less aggressive tumors. 


\section{RESULTS}

A

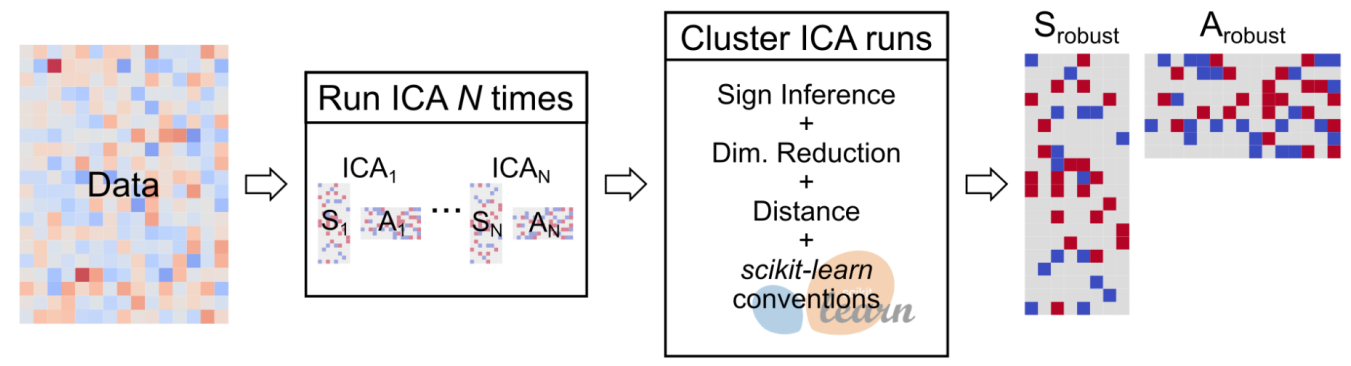

B

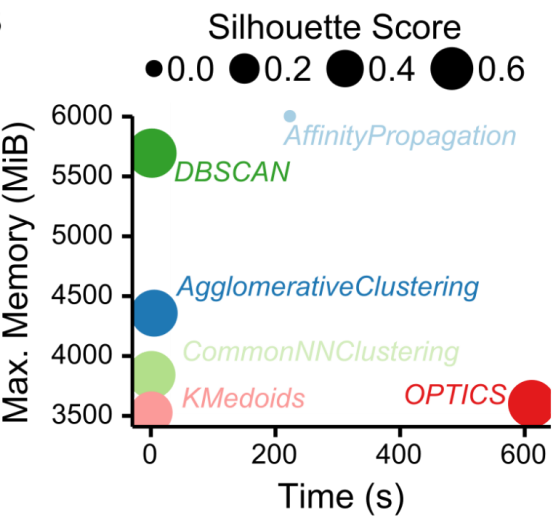

C

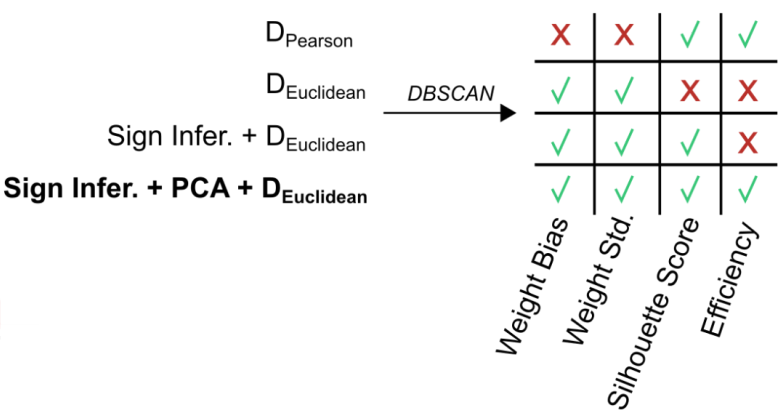

D

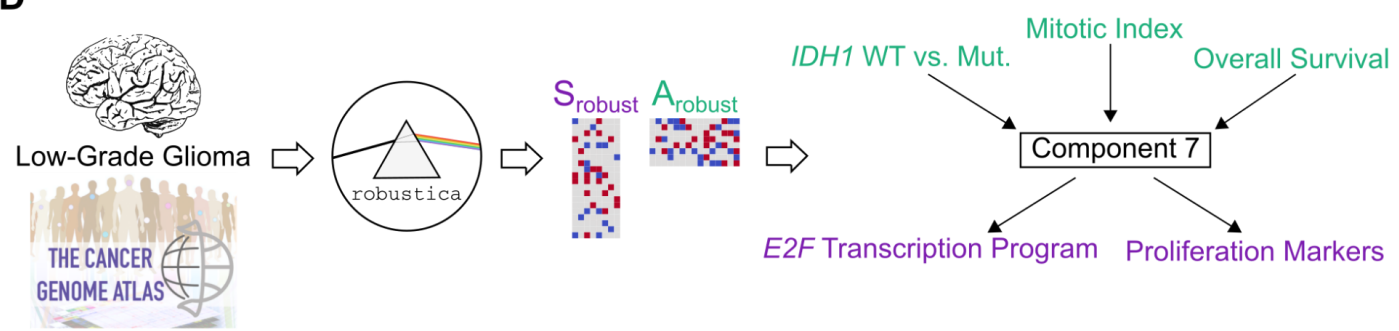

Figure 1. Development and implementation of robustica to carry out robust Independent Component Analysis (ICA).

(A) robustica enables fully customized robust ICA. We built robustica following scikit-learn's programming conventions to enable full control of both the iterative and clustering steps facilitating customization and optimization. In particular, robustica includes a subroutine to infer and correct the signs of components across ICA runs that improves the precision and efficiency of the clustering step by enabling us to use Euclidean distance metrics and to compress the feature space. (B) Comparison of clustering algorithms for robust ICA using Sastry $(2019)^{15}$ 's dataset. Maximum memory usage and time for each clustering algorithm to cluster 100 ICA runs with 100 components each. Dot sizes indicate median silhouette scores (the larger the better). (C) Development steps to improve the precision (Weight Std.) and efficiency while reducing the bias of robust ICA through our sign inference-and-correction subroutine combined with PCA and Euclidean distances, using Sastry (2019) $)^{15}$ 's dataset. (D) Case study workflow for robust ICA. We dissected $>500$ tumor samples from LGG patients with robustica into 100 robust independent components. Component 7 was simultaneously associated with multiple sample features (IDH1 mutation status, mitotic index, and overall survival) and contained genes known to be mechanistically associated with mutations in IDH1 that modulate tumor aggressiveness. 


\section{robustica enables systematic evaluation of clustering algorithms to perform robust ICA}

With robustica, one can fully customize the clustering method to use as long as they follow scikit-learn conventions ${ }^{14}$. We purposefully included this feature to compare how 6 different popular clustering algorithms perform at finding robust components (see Methods). As a benchmark, we dissected the $>250$ E. coli gene expression signatures from Sastry $(2019)^{15}$ into 100 components with 100 ICA runs and selected different clustering algorithms to compute the robust components. We then evaluated the performance of the different algorithms by measuring their run time, memory usage, and silhouette scores to quantify how similar each component is to the components in the cluster compared to the components assigned to other clusters. Overall, the DBSCAN algorithm showed the best performance taking $\sim 20$ seconds and $\sim 5500 \mathrm{MiB}$ of maximum memory usage to obtain clusters with the highest median silhouette scores (median=0.89). CommonNNClustering also performed well, with better memory efficiency at the cost of a lower silhouette score (median=0.81) (Fig. 1B; Sup. Fig. 2-3; Sup. Tab. 1).

\section{A}

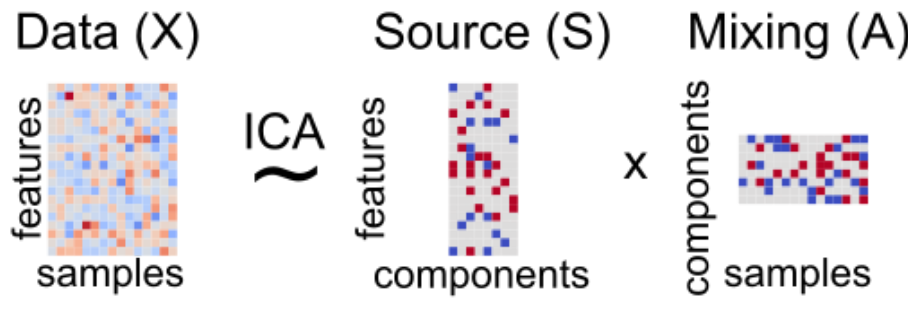

B

Icasso

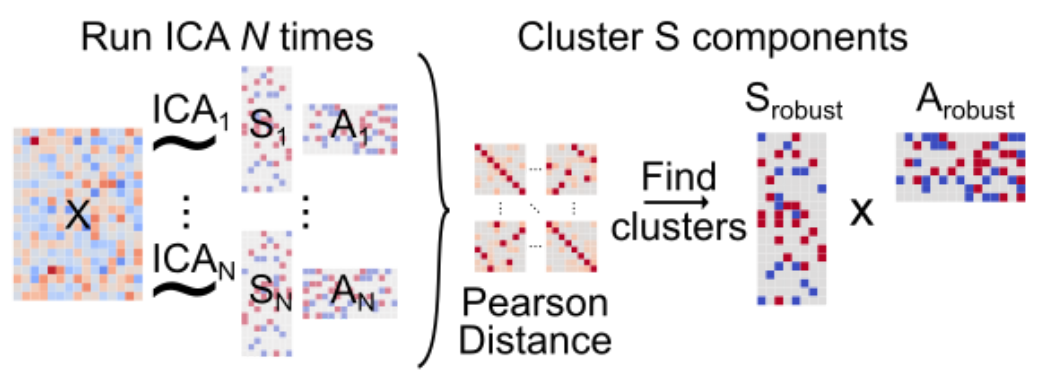

Supplementary Figure 1. Independent Component Analysis (ICA) and robust ICA with the Icasso algorithm.

(A) ICA is a matrix factorization algorithm applied for blind-source separation problems that decomposes a data matrix $(X)$ into $n$ independent components generating a source matrix $(S)$ and a mixing matrix $(A)$ with information on how features and samples contribute to each independent component, respectively. (B) The Icasso algorithm overcomes the inherent randomness of the Fast/CA algorithm -a widespread algorithm to perform ICA- by running ICA multiple times and clustering the resulting independent components in $S$ across all runs using an agglomerative clustering approach with average linkage and a Pearson distance matrix (n. components $\cdot n$. runs) $)^{2}$ dimensions as input. 

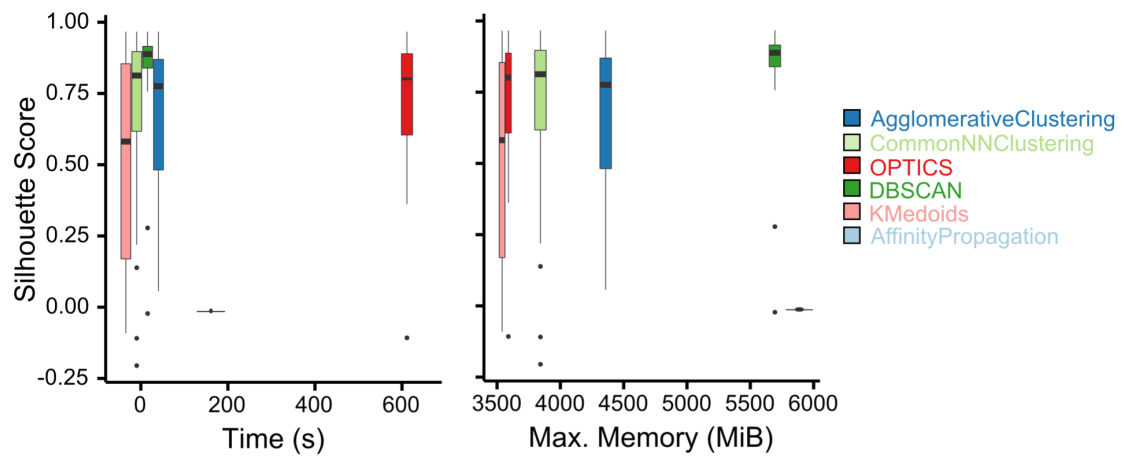

Supplementary Figure 2. DBSCAN shows the best performance to compute robust independent components.

Time (seconds) and maximum memory usage (MiB) compared to the average silhouette scores of each robust component (i.e. cluster) obtained using 6 different algorithms to cluster the independent components produced across 100 runs of ICA with $n$ _components $=100$ by dissecting Sastry $(2019)^{1}$ 's dataset.

$$
\begin{array}{rll} 
& -\bullet- & \text { cluster_components() } \\
\text { Function } & -\bullet- & \text { compute_centroids() } \\
-\bullet- & \text { compute_distance() }
\end{array}
$$
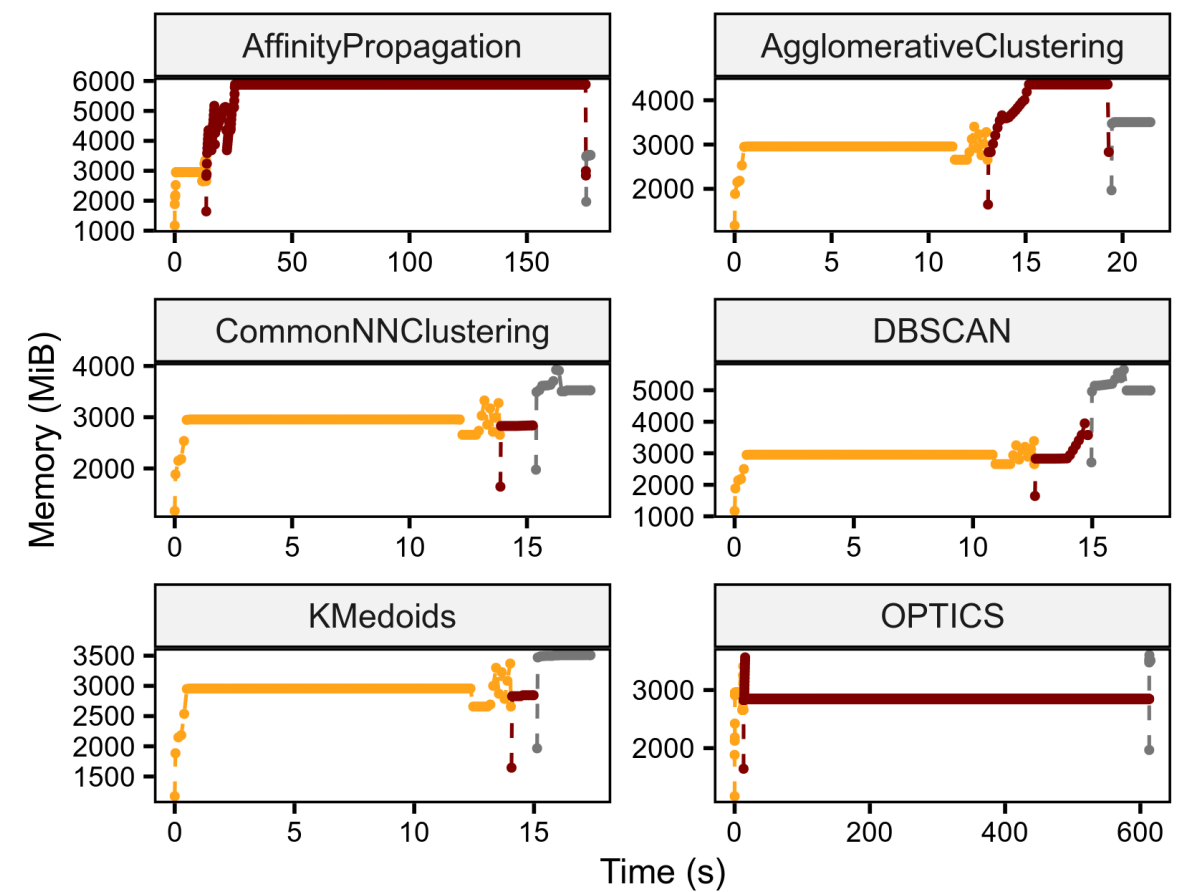

Supplementary Figure 3. Computing the Pearson distance matrix takes the most time when using the DBSCAN algorithm.

Memory usage across time and substeps (functions) to compute robust independent components in our comparison of clustering algorithms by dissecting Sastry (2019) $)^{1}$ 's dataset. 


\section{Clustering ICA runs with Euclidean distances improves the precision of robust ICA}

After running ICA multiple times, the Icasso algorithm computes a potentially large Pearson distance matrix (Sup. Fig. 1B). However, the sensitivity of Pearson distance to non-Gaussian distributions strongly biases weights in robust independent components, as the standard deviation of the weights across components in the same cluster strongly correlates with their average (Sup. Fig. 4-5). We tackled this problem with a simple subroutine to infer and correct the sign of the components across ICA runs to enable using Euclidean distances (see Methods). Our approach produced robust components with high silhouette scores and lowered the bias and standard deviation of their weights (Sup. Fig. 4-5). Since this implementation required less memory but more time to cluster sign-corrected components (Sup. Fig. 4D), we compressed the feature space of all ICA runs through PCA to reduce the overall run time and memory usage while maintaining the same performance (Sup. Fig. 4; Sup. Tab. 2-6).

Finally, we assessed how much the resulting gene modules differ using either Pearson or Euclidean distances (Sup. Tab. 7). While both approaches found highly similar modules, Euclidean distance tended to find more components of high silhouette scores (Sup. Fig. 6A and $\mathrm{B}$ ). In addition, we measured how much the high precision of Euclidean distance made the modules significantly more robust to random noise compared to Pearson distance and allowed using fewer runs to recover most of the gene modules defined using 100 ICA runs to compute robust components (Sup. Fig. 6C and D; Sup. Tab. 2-7). Bypassing the bias introduced by Pearson distances through Euclidean distances increased the reproducibility of the gene modules defined through robust ICA.

Our sign inference-and-correction subroutine creates high-quality, robust independent components by enabling us to efficiently cluster independent components across ICA runs using Euclidean distances (Fig. 1C). 
A

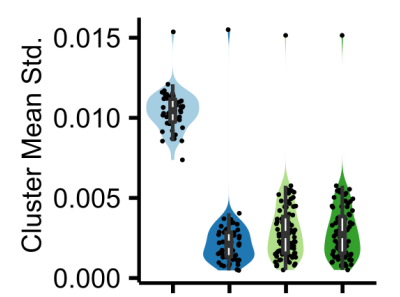

B

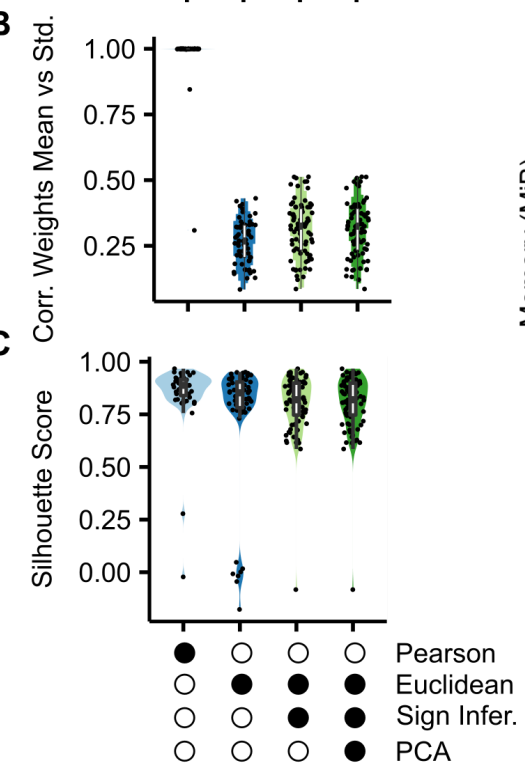

D
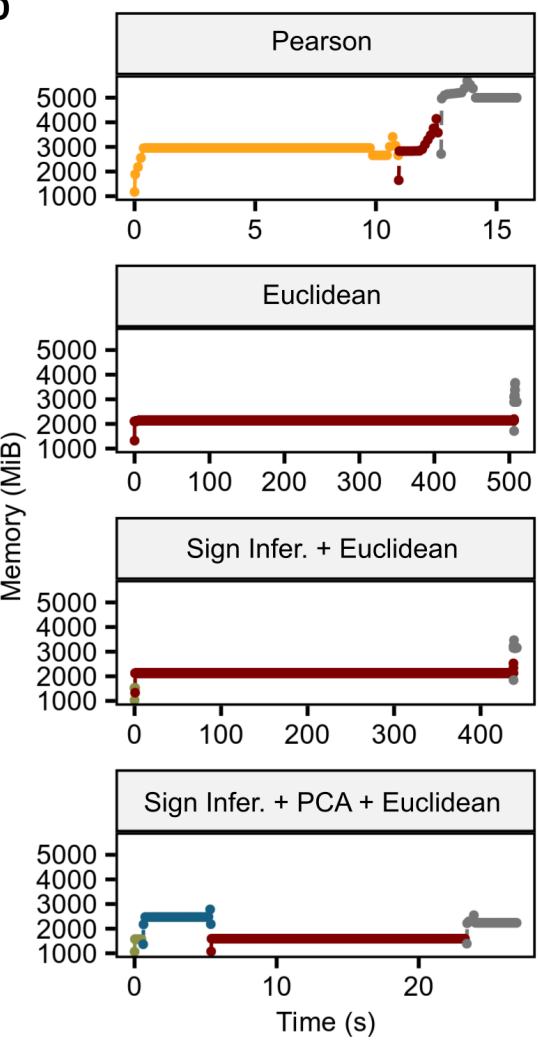

Supplementary Figure 4. Our sign inference-and-correction subroutine combined with PCA and DBSCAN with Euclidean distances leads to efficiently computing unbiased and precise robust independent components.

Each panel illustrates how our versions of the Icasso algorithm perform at dissecting Sastry (2019) 1 's dataset using: the original Pearson distance matrix (Pearson); plain Euclidean distance (Euclidean); Euclidean distance after our sign inference-and-correction subroutine (Sign Infer. + Euclidean); or Euclidean distance after our sign inference-and-correction subroutine and feature compression with PCA (Sign Infer. + PCA + Euclidean). (A) Average standard deviations of weights used to calculate the weights of each robust independent component. (B) Distributions of correlations between weight averages and weight standard deviations that correspond to each robust independent component. (C) Average silhouette scores of each component used to calculate the robust independent components. (D) Memory usage across time and substeps of every version of the Icasso algorithm: computing Pearson distance (yellow); clustering components with DBSCAN (dark red); computing centroids (grey); inferring components' signs (dark green); compressing feature space with PCA (dark blue). 


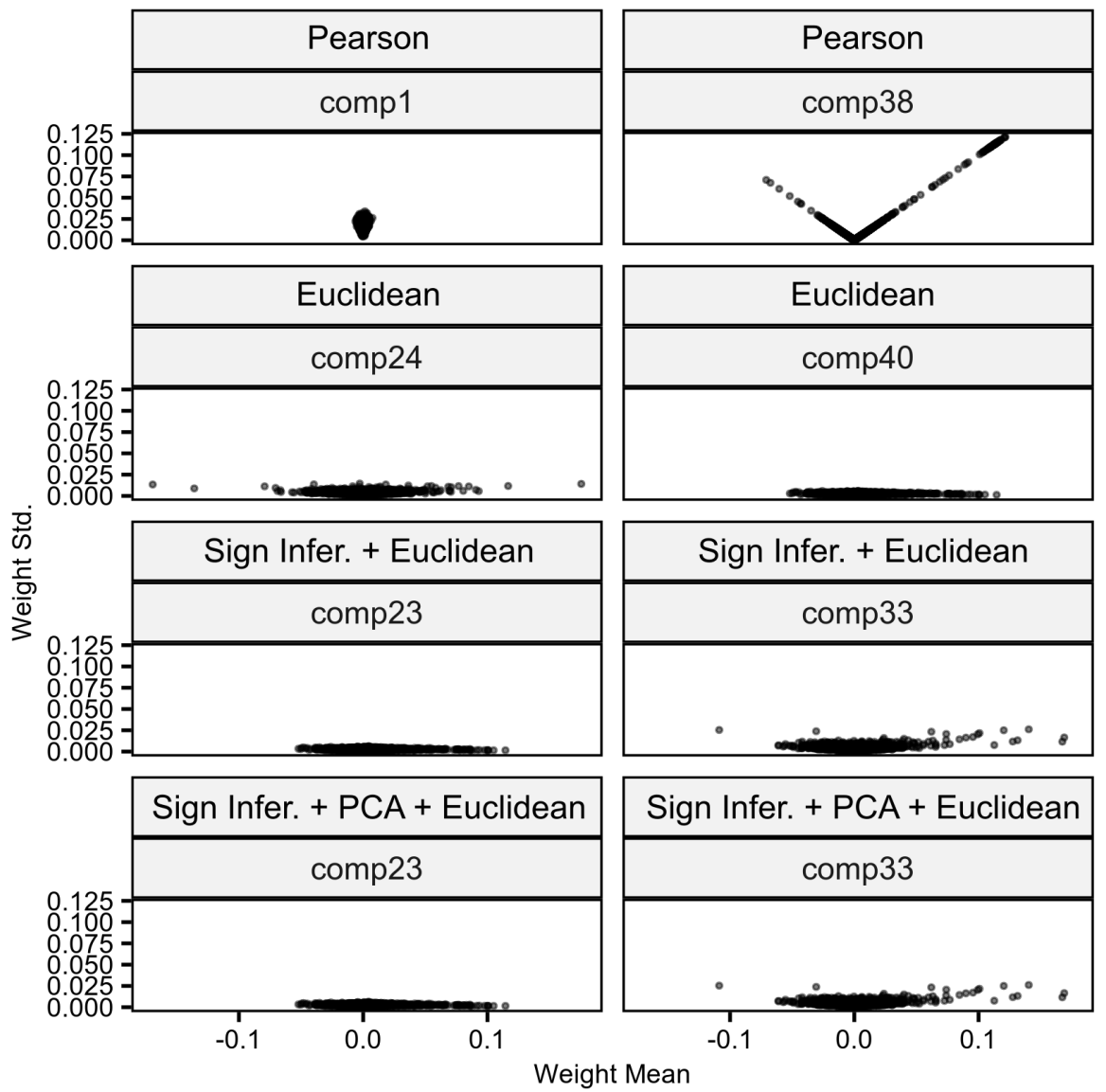

Supplementary Figure 5. Examples of weight mean and standard deviation bias in robust independent components.

The different panels illustrate how the mean and standard deviation of the components' weights used to compute robust independent components correlated depending on which version of the Icasso algorithm we used to dissect Sastry (2019)1 's dataset: the original Pearson distance matrix (Pearson); plain Euclidean distance (Euclidean); or Euclidean distance after our sign inference-and-correction subroutine (Sign Infer. + Euclidean); Euclidean distance after our sign inference-and-correction subroutine and feature compression with PCA (Sign Infer. + PCA + Euclidean). 

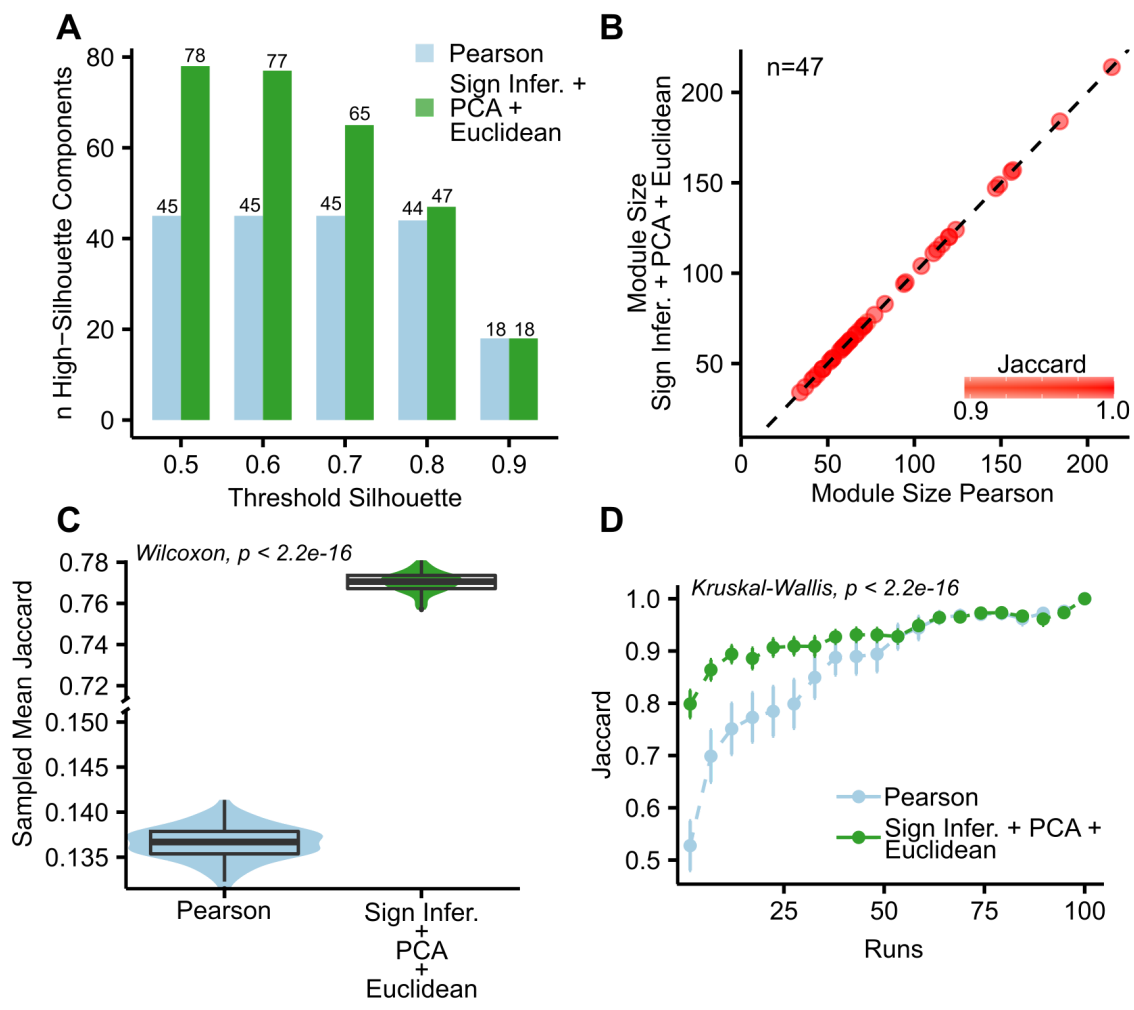

D

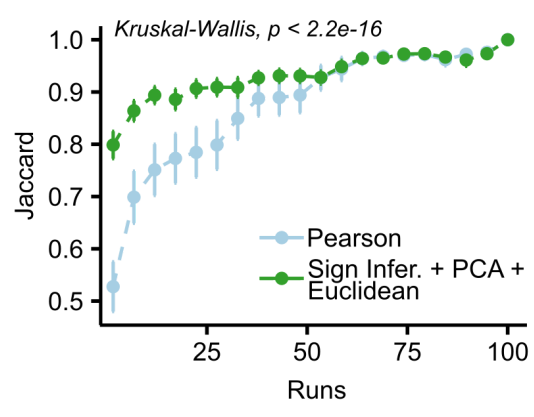

Supplementary Figure 6. Comparison between gene modules obtained from Sastry (2019) 1 's dataset with the classical (Pearson) and the revisited (Sign Infer. + PCA + Euclidean) Icasso algorithm to perform robust ICA.

(A) Number of components with high silhouette scores using different thresholds. (B) Sizes of gene modules uniquely mapped between the two approaches (47 in total). The color gradient indicates the degree of Jaccard similarity between mapped modules. (C) Average Jaccard similarities between the gene modules defined using inferred robust independent components and their corresponding weights subjected to random noise sampled 100 times. (D) Distributions (mean and standard deviation) of maximum Jaccard similarities between the gene modules defined using a different number of ICA runs compared with the gene modules defined using 100 ICA runs for both the classical and the revisited approaches. 


\section{robustica recovers a gene expression module with the key regulators of tumor aggressiveness in LGG mechanistically associated to mutations in IDH1}

As a case study, we dissected gene expression profiles from >500 LGG tumor samples from The Cancer Genome Atlas (TCGA). LGGs are characterized by mutations in the isocitrate dehydrogenase (IDH1) enzyme that decrease tumor aggressiveness by indirectly inhibiting the $E 2 F$ transcription program, an important switch controlling homeostasis and tumorigenesis ${ }^{16-18}$. We then explored whether certain gene modules associated with IDH1 mutations recovered known molecular mechanisms.

Weights in component 7 were highly associated with IDH1 mutation status, survival probability, and expression-based indices of cell proliferation (Sup. Fig. 7A-C). We related these sample traits to the gene expression signatures by defining a module of 421 genes (Sup. Fig. 7D; Sup. Tab. 8) which, as expected, was enriched in proliferation-related biological processes (Sup Fig. 8; Sup. Tab. 8) and contained 7 out of the 9 genes used to compute the mitotic index ${ }^{19}$ and known proliferation markers as $M K I 67^{20}$. Interestingly, our gene module also included $4 E 2 F$ transcription factors (E2F1, E2F2, E2F7, E2F8) and was enriched with 98 targets of E2Fs (Sup. Fig. 9; Sup. Tab. 8).

With this, we demonstrate the utility of robustica to identify gene sets whose combined expression in LGG is associated with genotypes and phenotypes of interest. Our identified module contained downstream effectors controlling cell proliferation associated with IDH1 mutation status and tumor aggressiveness (Fig. 1D), demonstrating the biological applicability of this approach. 

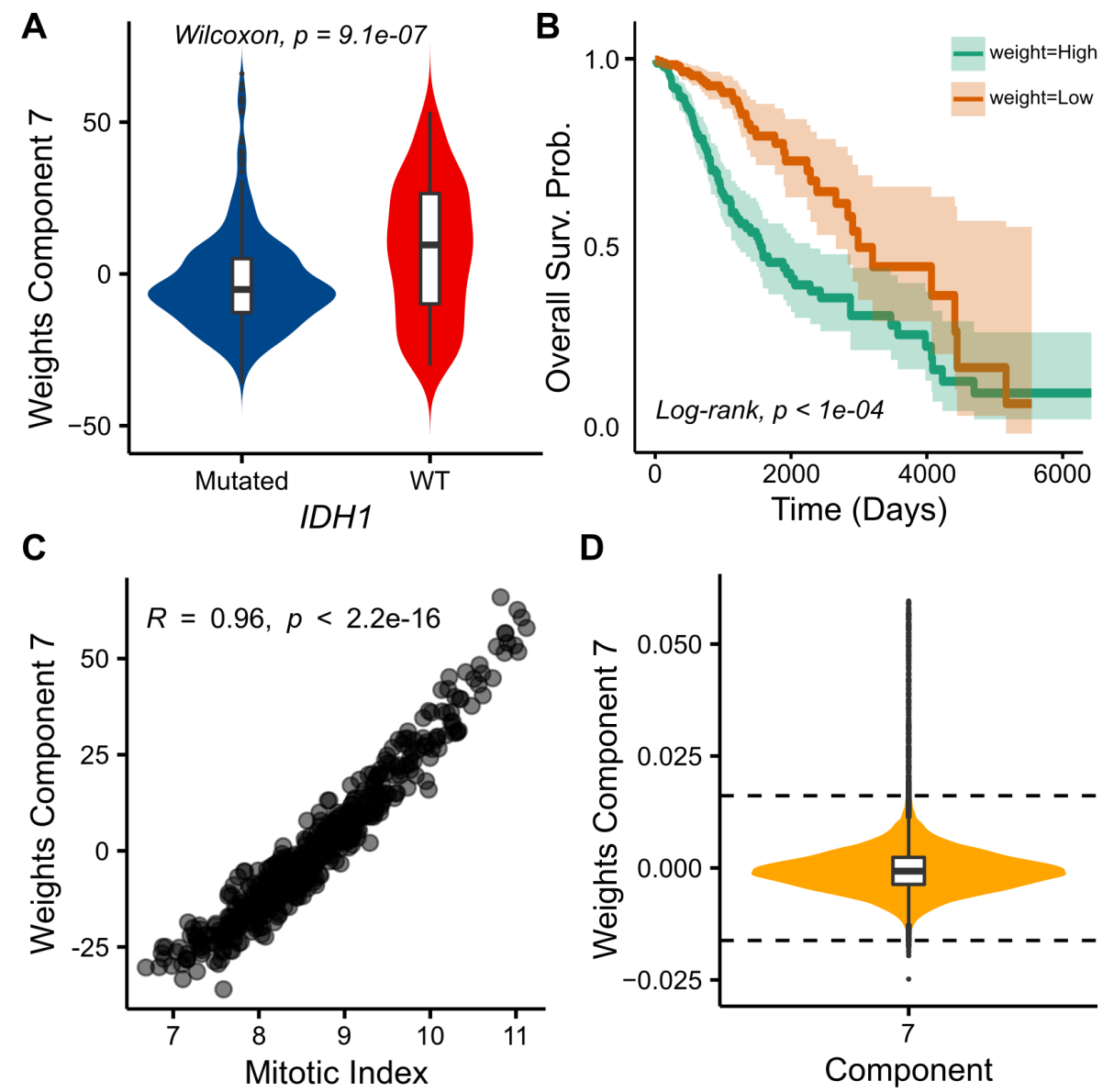

Supplementary Figure 7. Independent component 7 defines a module simultaneously associated with LGG patients' mutation status in IDH1, survival probability, and mitotic index.

(A) Distribution of sample weights in component 7 in the robust mixing matrix among patients with (Mutated) or without (WT) mutations in gene IDH1. We used a Wilcoxon Rank Sum test to assess the statistical differences between the groups (top label). (B) Kaplan-Meier curves of patients with weights higher or lower than the median weight in component 7 in the robust mixing matrix. The $p$-value of the log-rank test between the two groups is indicated at the bottom left. (C) Relationship between weights in component 7 in the robust mixing matrix and each sample's mitotic index. The Spearman correlation coefficient and its corresponding $p$-value are indicated at the top left ( $R$ and $p$, respectively). (D) Distribution of weights in component 7 in the robust source matrix used to define the gene module corresponding to our sample features of interest. The horizontal dashed lines indicate the thresholds applied to define the module. 


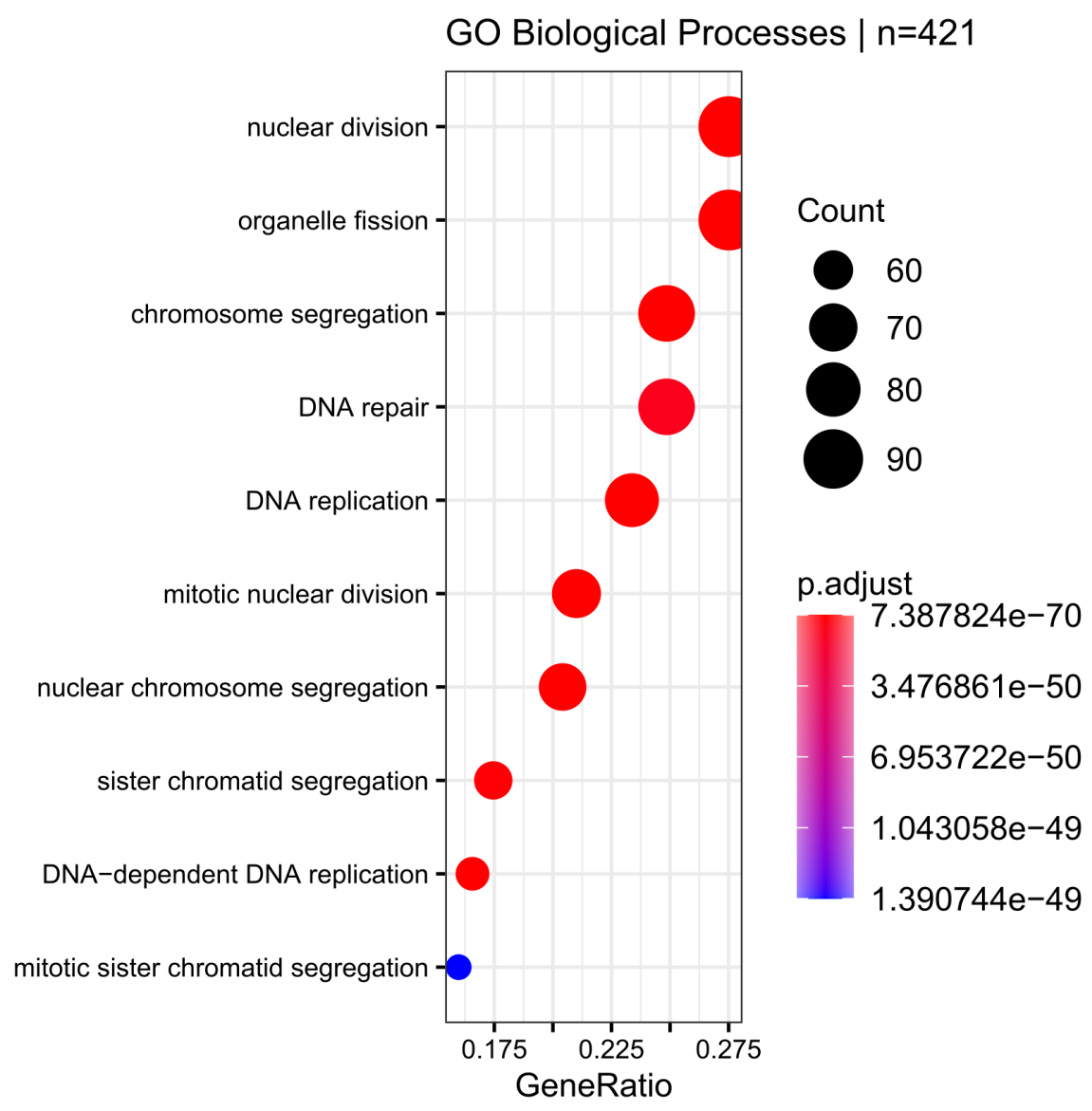

Supplementary Figure 8. The gene module defined from component 7 is enriched in proliferation-related biological processes.

Results from overrepresentation tests of our gene module defined from component 7 and Gene Ontology (GO) Biological Processes. 


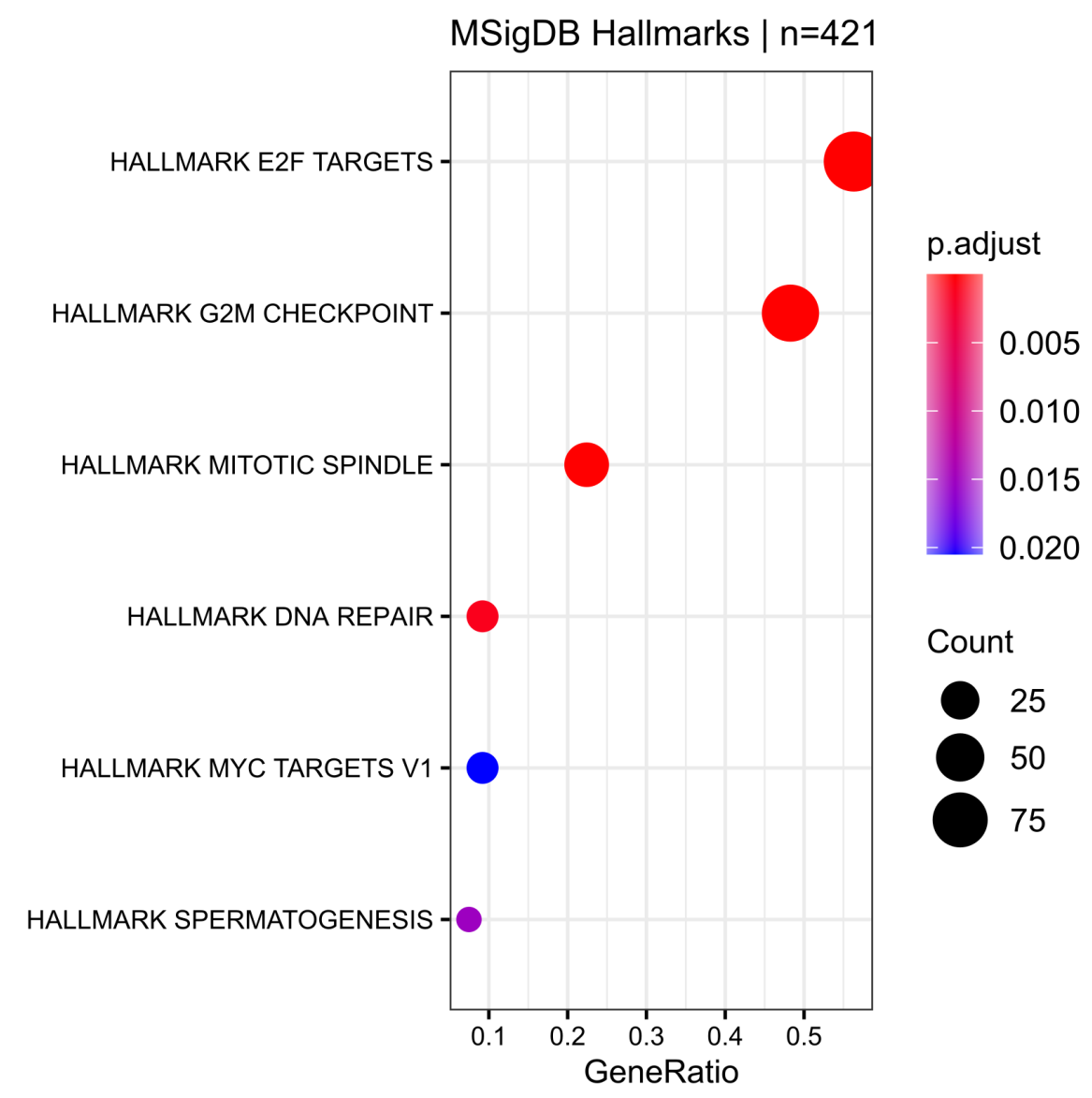

Supplementary Figure 9. The gene module defined from component 7 is enriched in targets of E2F transcription factors, known modulators of glioma aggressiveness.

Results from overrepresentation tests of our gene module defined from component 7 and MSigDB Hallmarks.

\section{CONCLUSION}

We created robustica, a new Python package built on top of scikit-learn that enables performing precise, efficient, and customizable robust ICA. Through its customizability, we explored how different clustering algorithms and distance metrics can further optimize robust ICA. Our sign correction subroutine improved the precision, robustness, and memory efficiency of the clustering step. Finally, we showcased how robustica can be used to explore gene modules associated with combinations of features of biological interest. Given the broad applicability of ICA for omic data analysis, we envision robustica will facilitate the seamless computation and integration of robust independent components in large pipelines.

\section{ACKNOWLEDGEMENTS}

We thank Xavier Hernandez-Alias, Marc Weber, and Leandro G. Radusky for the insightful discussions throughout the development of this project.

The results shown here are in part based upon data generated by the TCGA Research Network: https://www.cancer.gov/tcga. 


\section{FUNDING}

This project was funded in part by a grant from the Plan Estatal de Investigación Científica y Técnica y de Innovación to L.S. (PGC2018-101271-B-I00, http://www.ciencia.gob.es). We also acknowledge the support of the Spanish Ministry of Science and Innovation to the EMBL partnership, the Centro de Excelencia Severo Ochoa, and the CERCA Programme / Generalitat de Catalunya.

\section{COMPETING INTERESTS}

The authors have declared that no competing interests exist. 


\section{REFERENCES}

1. HERAULT, J. \& ANS, B. Réseau de neurones à synapses modifiables: décodage de messages sensoriels composites par apprentissage non supervisé et permanent. Réseau Neurones À Synap. Modif. Décodage Messag. Sensoriels Compos. Par Apprentiss. Non Supervisé Perm. 299, 525-528 (1984).

2. Sompairac, N. et al. Independent Component Analysis for Unraveling the Complexity of Cancer Omics Datasets. Int. J. Mol. Sci. 20, (2019).

3. Liebermeister, $W$. Linear modes of gene expression determined by independent component analysis. Bioinformatics 18, 51-60 (2002).

4. Lee, S.-I. \& Batzoglou, S. Application of independent component analysis to microarrays. Genome Biol. 4, R76 (2003).

5. Stein-O'Brien, G. L. et al. Enter the Matrix: Factorization Uncovers Knowledge from Omics. Trends Genet. 34, 790-805 (2018).

6. Cantini, L. et al. Assessing reproducibility of matrix factorization methods in independent transcriptomes. Bioinformatics 35, 4307-4313 (2019).

7. Way, G. P., Zietz, M., Rubinetti, V., Himmelstein, D. S. \& Greene, C. S. Sequential compression of gene expression across dimensionalities and methods reveals no single best method or dimensionality. bioRxiv 573782 (2019) doi:10.1101/573782.

8. Hyvärinen, A. \& Oja, E. A Fast Fixed-Point Algorithm for Independent Component Analysis. Neural Comput. 9, 1483-1492 (1997).

9. Himberg, J. \& Hyvarinen, A. Icasso: software for investigating the reliability of ICA estimates by clustering and visualization. in 2003 IEEE XIII Workshop on Neural Networks for Signal Processing (IEEE Cat. No.03TH8718) 259-268 (2003). doi:10.1109/NNSP.2003.1318025.

10. Biton, A. Mine/CA: Analysis of an ICA decomposition obtained on genomics data. (Bioconductor version: Release (3.13), 2021). doi:10.18129/B9.bioc.MinelCA.

11. LabBandSB/BIODICA: 'Independent Component Analysis of Blg Omics Data'. GitHub https://github.com/LabBandSB/BIODICA.

12. Zheng, C.-H., Huang, D.-S., Kong, X.-Z. \& Zhao, X.-M. Gene Expression Data Classification Using Consensus Independent Component Analysis. Genomics Proteomics Bioinformatics 6, 74-82 (2008).

13. Jiang, D., Tang, C. \& Zhang, A. Cluster analysis for gene expression data: a survey. IEEE Trans. Knowl. Data Eng. 16, 1370-1386 (2004).

14. Pedregosa, F. et al. Scikit-learn: Machine Learning in Python. J. Mach. Learn. Res. 12, 2825-2830 (2011).

15. Sastry, A. V. et al. The Escherichia coli transcriptome mostly consists of independently regulated modules. Nat. Commun. 10, 5536 (2019).

16. Miyata, S. et al. An R132H Mutation in Isocitrate Dehydrogenase 1 Enhances p21 Expression and Inhibits Phosphorylation of Retinoblastoma Protein in Glioma Cells. Neurol. Med. Chir. (Tokyo) 53, 645-654 (2013).

17. Youssef, G. \& Miller, J. J. Lower Grade Gliomas. Curr. Neurol. Neurosci. Rep. 20, 21 (2020).

18. Fang, Z. H. \& Han, Z. C. The transcription factor E2F: a crucial switch in the control of homeostasis and tumorigenesis. Histol. Histopathol. 403-413 (2006) doi:10.14670/HH-21.403.

19. Yang, Z. et al. Correlation of an epigenetic mitotic clock with cancer risk. Genome Biol. 17, 205 (2016). 
bioRxiv preprint doi: https://doi.org/10.1101/2021.12.10.471891; this version posted January $13,2022$. The copyright holder for this

preprint (which was not certified by peer review) is the author/funder, who has granted bioRxiv a license to display the preprint in perpetuity. It is made available under aCC-BY-NC-ND 4.0 International license.

20. Gerdes, J. et al. Cell cycle analysis of a cell proliferation-associated human nuclear antigen defined by the monoclonal antibody Ki-67. J. Immunol. 133, 1710-1715 (1984). 